Western University

Scholarship@Western

Department of Economics Research Reports

Economics Working Papers Archive

1973

\title{
The Aggregate Excess Demand Correspondence and the Structure of Economies with Externalities
}

David T. Scheffman

Follow this and additional works at: https://ir.lib.uwo.ca/economicsresrpt

Part of the Economics Commons

Citation of this paper:

Scheffman, David T.. "The Aggregate Excess Demand Correspondence and the Structure of Economies with Externalities."

Department of Economics Research Reports, 7325. London, ON: Department of Economics, University of Western Ontario (1973). 
Research Report 7325

THE AGGREGATE EXCESS DEMAND CORRESPONDENCE AND THE STRUCTURE OF ECONOMIES WITH EXTERNALITIES

by

D. T. Scheffman 
The Aggregate Excess Demand Correspondence and the Structure of Economies with Externalities

\author{
D. T. Scheffman \\ University of Western Ontario*
}

In this paper will be concerned with two problems: a) in an economy with externalities, what are the properties of the feasible set of consumption and production vectors? and b) what would we mean by the aggregate excess demand correspondence in an economy with externalities, and what are its properties? Most of problem (a) has been adequately solved in the recent paper by 0 sana, $^{1}$ but our analysis will enlarge somewhat on his results because both our model and methods of proof are somewhat different from his. Our solution to (a) and (b) will allow us to directly compare certain properties of general equilibrium models with and without externalities. The class of economies with externalities we shall consider will be a generalization of the class considered by Arrow and Hahn. ${ }^{2}$ Existence of competitive equilibrium for the class of economies we shall consider has been established elsewhere by the author. ${ }^{3}$

To our knowledge, problem (b) has heretofore not been considered. To see what is involved, consider a simple two person, two good, pure exchange economy in which consumption externalities are present. Let the utility function of the two consumers be $U^{1}\left(x_{1}^{1}, x_{2}^{1} ; x_{1}^{2}, x_{2}^{2}\right) U^{2}\left(x_{1}^{2}, x_{2}^{2} ; x_{1}^{1}, x_{2}^{1}\right)$ (here superscripts index the consumers, subscripts index the goods). The excess demand functions (for simplicity here will assume single-valued demand) of each of the consumers are functions not only of prices $\left(p_{1}, p_{2}\right)$, but of the consumption of the other consumer. Thus if $f_{1}^{1}$ is the excess demand 
function of consumer one, $f_{1}^{1}=f_{1}^{1}\left(p_{1}, p_{2} ; x_{1}^{2}, x_{2}^{2}\right)$. (We will call $\left(x_{1}^{2}, x_{2}^{2}\right)$ here the "reference" consumption bundle for consumer one).

The interpretation of $\mathrm{f}_{1}^{1}$ is not clear without imposing further structure on the model--i.e., is $\left(x_{1}^{2}, x_{2}^{2}\right)$ actual consumption, or predicted consumption, or what? One possibility is to take an extreme Walrasian viewpoint that in this case the auctioneer calls out not only prices, but also reference consumption bundles. It is not the purpose of this paper to consider this particular problem, so we will only briefly allude to the problem involved at relevant points of our analysis.

In the usual general equilibrium models without externalities, the $i^{\text {th }}$ person's excess demand vector is a function only of prices, i.e., $f^{i}=f^{i}(p)$, and we can simply add these excess demand correspondences, $f(p)=\Sigma f^{i}(p)$, to get the aggregate excess demand correspondence. As we have already noted, once externalities are present, the individual excess demand correspondences are defined not only on prices, but also on other person's consumptions (and possibly, as our model will allow, on production decisions). Therefore if we attempted to create the aggregate excess demand correspondences by simply adding the individual correspondences, the aggregated would be defined not only on prices, but on the "reference" consumption (and production) points in each individual correspondence. (In terms of our two person, two good, pure exchange economy, $f_{1}=f_{1}^{1}\left(p_{1}, p_{2} ; x_{1}^{2}, x_{2}^{2}\right)+f_{1}^{2}\left(p_{1}, p_{2} ; x_{2}^{1}, x_{2}^{2}\right)$, so that $f_{1}=f_{1}\left(p_{1}, p_{2} ; x_{1}^{1}, x_{2}^{1} ; x_{1}^{2}, x_{2}^{2}\right)$. We will present an alternative definition of the aggregate excess demand correspondence, in which aggregate demand, so defined, is a function only of prices. Our defined aggregate correspondence is then similar to the aggregate correspondence for a model without externalities. Thus by examining 
the properties of our defined correspondence we can examine what the effects of externalities are on static Walrasian models. Our solutions of problems (a) and (b) will show that in a sense, externalities introduce non-convexities into such models.

A: $\quad$ The Mode1

The class of externalities allowable within the framework of the model we shall consider includes most of the types considered in the 1iterature. These include:

a. Pure consumption externalities: a consumer's consumption may affect the preferences and/or the consumption possibilities of other consumers.

b. Pure production externalities: a producer's production activity may affect the production possibilities of other producers.

c. Production-consumption externalities: a producer's production activity may affect the preferences and/or consumption possibilities of consumers.

d. Consumption-production externalities : a consumer's consumption may affect the production possibilities of producers.

Externalities of type (c) or (d) have not had much emphasis in the 1iterature, although those of type (c) would seem to be an important real world phenomenon. We assume that all externalities are identifiable with the set of tradeable goods in the model. 
As a basis for comparison we shall briefly review the general equilibrium model found in most of the conventional existence 1iterature. In that model there are a fixed number of goods numbered $h=1, \ldots, n$; a fixed number of consumers numbered $i=1, \ldots, s$; and a fixed number of producers $e=1, \ldots, t$

A consumer, $i$, is a consumption possibilities set, $x^{i} \in R^{n}$, a set of preferences $(\geqq)_{i}$ defined on $x^{i}$, and an initial endowment $w^{i} \in R$. It is assumed that $x^{i}$ is closed, bounded from below, and convex; that $(\geqq)_{i}$ is continuous and convex; $4 \bar{x}^{i} \in x^{i}$ such that $\bar{x}^{i} \ll w^{i}$.

A producer, e, is a net production possibilities set $Y^{e} \in R^{n} . Y^{e}$ is assumed to be closed and convex, and $0 \in \mathrm{Y}^{\mathrm{e}} \cdot \mathrm{y}^{\mathrm{e}} \in \mathrm{Y}^{\mathrm{e}}$ is a net input-output vector.

The mode1 we consider has:

$$
\begin{array}{ll}
\text { n goods, } & h=1, \ldots, n \\
\text { s consumers, } & i=1, \ldots, s \\
t \text { producers, } & e=1, \ldots, t
\end{array}
$$

An action for consumer $i$ is the choice of a consumption vector $x^{1} \in R^{n}$. An action for producer $e$ is the choice of an input and associated output vector $q^{e}=\left(q^{I e}, q^{0 e}\right) \in R^{2 n}$ where $q^{\text {Ie }}$ is an input vector and $q^{0 e}$ an associated output vector. We depart from the usual treatment of production by disaggregation into inputs and outputs rather than considering only net outputs. The reason for this is that intermediate goods would seem to be important in conveying externalities. For example, if a coal mine produces an externality (coal dust) which affects a nearby laundry, coal produced and used as an intermediate good in the production of coal (e.g., burned) will also have an external effect. of course the actions 
of consumer $i$ are limited by his consumption possibilities and the actions of producer e are limited by his production possibilities, both of which we shall define in the following sections. A set of proposed actions by the participants of the model is denoted $(z, y) \in R^{(s+2 t) n}$ where $z=\left(x^{1}, \ldots, x^{s}\right)$ and $y=\left(q^{1}, \ldots, q^{t}\right)$.

\section{A-1: Consumers}

A consumer, $i$, has a consumption possibilities correspondence which for each vector of actions of other consumers and producers defines the consumption possibilities of $i$. This correspondence will be denoted $x^{i}(z, y)$ where $z=\left(x^{1}, \ldots, x^{s}\right), y=\left(q^{1}, \ldots, q^{t}\right)$. Thus $x^{i}: R^{(s+2 t) n} \rightarrow R^{n} \cdot x^{i}(z, y)$ defines the set of consumptions for $i$ given $(z, y)$ which allow him to survive. This is the main difference between our class of economies and the class considered by Arrow and Hahn. In their analysis they assumed the consumption sets were fixed.

Assumptions about $x^{i}(z, y)$ :

1) $x^{i}(z, y)$ does not depend on $x^{i}$, and is closed, convex, uniformly bounded from below, and continuous in $(z, y)$, for all $(z, y) \in R^{(s+2 t) n} \cdot\left(x^{i}(z, y)\right.$ is a correspondence, not a function, and thus by continuity we mean both upper and lower semi-continuous).

2) Each consumer, $i$, has an initial endowment function, $w^{i}(z, y), w^{i}: R^{(s+2 t) n} \rightarrow R^{n}$ which is continuous in $(z, y)$, and $w^{i}(z, y) \geqq 0$, for all $(z, y) \in R^{(s+2 t) n}$. $\pi^{-i}$ such that $w^{i}(z, y) \leqq w$, for all $(z, y)$ and $\forall(z, y)$ B $\bar{x}^{i}(z, y) \in x^{i}(z, y)$ such that $\bar{x}^{i}(z, y) \ll w^{i}(z, y)$. 
Comments: That $x^{i}(z, y)$ is closed, convex and uniformly bounded from below, for all $(z, y)$ is the obvious generalization of the assumptions made about the (fixed) consumption set in the usual existence problem. However, the assumption of continuity of $\mathrm{x}^{i}(z, y)$ is a very strong assumption. Upper semi-continuity of $x^{i}(z, y)$ would be a more acceptable assumption, but unfortunately it is not strong enough to establish existence of equilibrium. That $w^{i}(z, y)$ be bounded seems to be a reasonable assumption. As mentioned In our brief summary of the assumptions for economies without externalities, the usual assumption is that $\exists \bar{x}^{-i} \in x^{i}$ such that $\bar{x}^{i} \ll w^{i}$. Our assumption in (2) is analogous. We could considerably weaken the assumption that $\bar{x}^{i}(z, y) \ll w^{i}(z, y)$ in a manner analogous to that of Debreu, ${ }^{4}$ but only at the cost of a serious loss of clarity of exposition, and for this reason we will use the stronger assumption.

A consumer, $i$, has a preference relation $(\geqq)_{i}$ defined on $\left\{(z, y, p) \mid z \in R^{s n}, y \in R^{2 t n}, p \in R^{n}\right\}$. Here $z=\left(x^{1}, \ldots, x^{s}\right)$ is a vector of all consumers' actions, $y=\left(q^{1}, \ldots, q^{t}\right)$ is a vector of all producers' actions, and $p=\left(p_{1}, \ldots, p_{n}\right)$ is a price vector. We allow consumers' preferences to be defined on prices also since it includes a model discussed in the literature and its inclusion causes no extra difficulties. Assumptions about $(\geqq)_{i}$ :

3) ( $)_{i}$ is a complete continuous preorder on $R^{(s+2 t+1) n}$. By continuous we mean the sets:

$$
\left\{(z, y, p) \in R^{(s+2 t+1) n} \mid(z, y, p)(\geqq)_{i}(\bar{z}, \bar{y}, \bar{p})\right\}
$$

and

$$
\left\{(z, y, p) \in R^{(s+2 t+1) n} \mid(\bar{z}, \bar{y}, \bar{p})(\geqq)_{i}(z, y, p)\right\}
$$

are closed in $R^{(s+2 t+1) n}$, for all $(\bar{z}, \bar{y}, \bar{p}) \in R^{(s+2 t+1) n}$. 
4) Let $(z, y, p)=\left(\left(x^{1}, \ldots, x^{i-1}, x^{i}, x^{i+1}, \ldots, x^{s}\right), y, p\right)$

and $(\bar{z}, y, p)=\left(\left(x^{1}, \ldots, x^{i-1}, \bar{x}^{i}, x^{i+1}, \ldots, x^{s}\right), y, p\right)$ be

points in $R^{(s+2 t+1) n}$ and $(z, y, p)(>)_{i}(\vec{z}, y, p)$. Then

$k(z, y, p)+(1-k)(\bar{z}, y, p)(>)_{i}(\bar{z}, y, p)$, for all $k \in(0,1)$.

Comments: The continuity of preferences we assume here is analogous to the continuity of preferences assumption made in the usual case. It rules out preferences such as lexicographic orderings. With externalities however continuity of preferences is probably a somewhat less reasonable assumption. For example, I may like my brother-in-law to have more, but I do not like him to have as much as I do. This sort of preference structure would be discontinuous. The convexity assumption in (4) is completely analogous to the usual convexity assumption. Notice it requires convexity of preference only in a consumer's own consumption, given fixed actions of other participants.

Notation: Let $z_{i}=$ the closed convex hull of the $\operatorname{set}\left\{x^{i} \mid x^{i} \in x^{i}(z, y)\right.$ for some $(z, y)\}$. Let $z={\underset{i=1}{X}}_{i} z_{i}$ and let $P=\left\{p \in R^{n} \mid \sum_{h=1}^{n} p_{h}=1, p_{h} \geqq 0\right\}$. The production correspondence of the $e^{\text {th }}$ producer will be denoted $Y^{e}(z, y)\left(Y^{e}: R^{(s+2 t) n} \rightarrow R^{2 n}\right)$. Let $Y_{e}=$ the closed convex hull of the set $\left\{y^{e} \mid y^{e} \in Y^{e}(z, y)\right.$ for some $\left.(z, y)\right\}$. Let $Y=\underset{e=1}{t} Y_{e} \cdot$ Finally let $W=Z \times Y \times P$. For

$$
z \in z, z=\left(x^{1}, \ldots, x^{s}\right)
$$

let $z_{i}=x^{i}$, and

$$
i_{z}=\left(x^{1}, \ldots, x^{i-1}, x^{i+1}, \ldots, x^{s}\right) .
$$


Definition: Let $B^{i}(z, y, p)=\left\{x^{i} \in x^{i}(z, y) \mid p \cdot x^{i}-w^{i}(z, y) \leqq v^{i}(z, y, p)\right\}$ where $v^{i}(z, y, p)$ is a continuous non-negative real-valued function on $w$. $B^{i}(z, y, p)$ is the budget correspondence for consumer $i$. Thus for each $(z, y, p) \in W, B^{i}(z, y, p)$ is the set of possible actions for consumer $i$ which are both technically and economically feasible for consumer $i . v^{i}(z, y, p)$ will eventually be shown to be the share of profits function for consumer $i$. Definition: The demand correspondence of consumer $i, f^{i}: W \rightarrow z_{i}$ is defined

$$
\begin{gathered}
f^{i}(z, y, p)=\left\{\bar{x}^{i} \in B^{i}(z, y, p) \mid(\bar{z}, y, p)(\geqq){ }_{i}\left(z^{\prime}, y, p\right),\right. \\
\text { for all } \bar{z}, z^{\prime} \text { such that } \bar{z}_{i}=\bar{x}^{i}, \\
\left.i \bar{z}={ }^{i} z^{\prime}={ }^{i} z \text {, and } z_{i}^{\prime} e B^{i}(z, y, p)\right\}
\end{gathered}
$$

Theorem I: Under assumptions (1)-(4) and assuming $W$ is compact, $f^{1}$ is an upper semi-continuous, convex-valued correspondence.

We will not prove this theorem here since it is a relatively straightforward exercise. In the proof, first the continuity of the budget correspondence $\mathrm{B}^{i}$ is established, from which it easily follows that $f^{i}$ is upper semi-continuous (u.s.c.). The convexity of $f^{i}$ follows directly from the convexity of $x^{i}(z, y)$ and $(\geqq)_{i}$.

Theorem I establishes that in models with externalities, consumers' excess demand correspondences are u.s.c. and convex-valued just as in models without externalities, but of course with externalities the demand correspondence has more arguments than prices. 


\section{A-2: $\quad$ Producers}

A producer, $e,(e=1, \ldots, t)$ has a production possibilities correspondence $Y^{e}: R^{(s+2 t) n} \rightarrow R^{2 n}$ denoted $Y^{e}(z, y)$. A typical element of the range of $Y^{e}(z, y)$ is $q^{e}=\left(q^{I e}, q^{0 e}\right) \in R^{2 n} . q^{e} \in Y^{e}(z, y)$ means that if $(z, y)$ represents the actions of all other participants then $q^{e}=\left(q^{I e}, q^{\text {Oe }}\right)$ is technologically feasible input-output combination for producer e. It is assumed that $\mathrm{Y}^{\mathrm{e}}(\mathrm{z}, \mathrm{y})$ does not depend on $\mathrm{y}_{\mathrm{e}}=q^{\mathrm{e}}$.

Notation: For $y=\left(q^{1}, \ldots, q^{t}\right)$, let $y_{e}=q^{e}$

$$
e_{y}=\left(q^{1}, \ldots, q^{e-1}, q^{e+1}, \ldots, q^{t}\right) \text {, }
$$

and $\quad \hat{y}_{e}=q^{O e}-q^{I e}=\hat{q}^{e}$

$\hat{y}_{e}\left(=\hat{q}^{e}\right)$ is just the net output vector corresponding to the input-output combination $y_{e}=q^{e}=\left(q^{I e}, q^{0 e}\right)$. Similarly define $\hat{Y}^{e}(z, y)=\left\{\hat{q}^{e} \mid q^{e} \in \mathrm{Y}^{e}(z, y)\right\}$. Then $\hat{\mathrm{Y}}^{e}(z, y)$ is the net output correspondence of producer $e$.

Assumptions about $\mathrm{Y}^{\mathrm{e}}(\mathrm{z}, \mathrm{y})$ :

5) $\mathrm{Y}^{\mathrm{e}}(\mathrm{z}, \mathrm{y})$ is a continuous convex-valued correspondence. (This implies $\mathrm{Y}^{\mathrm{e}}(\mathrm{z}, \mathrm{y})$ is closed, for a11 $\left.(z, y)\right)$.

6) $0 \in Y^{e}(z, y)$, for $a 11(z, y)$

\section{Comments:}

That $\mathrm{Y}^{\mathrm{e}}(z, y)$ is closed and convex for all $(z, y)$ is analogous to the usual assumption made about production sets in the no externalities case. This means that given fixed actions of other participants, producer $e^{\prime} s$ production possibilities do not exhibit indivisibilities or increasing returns. This however is perfectly consistent with aggregate increasing returns resulting from externalities between producers. In this case, however, it is necessary to interpret a single producer as a firm rather 
than as an industry. Our assumption that $\mathrm{Y}^{\mathrm{e}}(\mathrm{z}, \mathrm{y})$ be a continuous correspondence is very strong, but it is consistent with most treatments of production externalities found in the literature, where an individual producer is assumed to have a continuous (and usually differentiable) production function which has as its arguments the producer's inputs, and the actions of other producers.

Again, we will not prove the following Theorem and Corollary, since their proofs are fairly standard exercises.

Definition: The supply correspondence of producer $e, r^{e}: W \rightarrow Y_{e}$ is defined

$$
\begin{aligned}
& r^{e}(z, y, p)=\left\{\bar{q}^{e} \dot{\varepsilon} Y^{e}(z, y) \mid p \cdot \hat{\bar{q}}^{e} \geqq p \cdot \hat{q}^{e},\right. \\
& \text { for all } \left.q^{e} \in Y^{e}(z, y)\right\} \text {. }
\end{aligned}
$$

Theorem II: Under assumption (5) and assuming $W$ is $\operatorname{compact} r^{e}(z, y, p)$ is an upper semi-continuous convex-valued correspondence.

Definition: We define the profit function of producer $e, \pi^{e}(z, y, p)$ as:

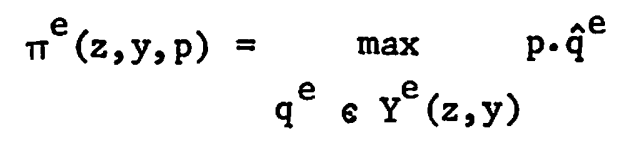

By the assumption that $W$ is compact, $\pi^{e}(z, y, p)$ is well defined. (Clearly, $\pi^{e}(z, y, p)$ is single-valued $)$.

The following obvious corollary is stated without proof:

Corollary: $\pi^{e}(z, y, p)$ is continuous.

Assumption: Each consumer, $i$, owns a proportion $d_{e}^{i}$ of the $e^{\text {th }}$ firm.

$\sum_{i=1}^{s} d_{e}^{i}=1, d_{e}^{i} \in[0,1]$, for all $i, e$.

Definition: Let $v^{i}(z, y, p)$ used in the definition of $B^{i}(z, y, p)$ be defined

$$
v^{i}(z, y, p)=\sum_{e=1}^{t} d^{i} \cdot \pi^{e}(z, y, p) .
$$

By the Corollary, $v^{i}(z, y, p)$ is continuous and by $(6) v^{i}(z, y, p) \geqq 0$. 
We have now completely described consumers, producers and their actions for a compact economy. In the next section we show how a general economy can be "compactified" so that the assumptions of compactness of $Z_{i}$ and $Y_{e}$ are satisfied.

We return now to the problem of how we interpret the correspondences $f^{i}(z, y, p)$ and $r^{e}(z, y, p)$. As mentioned at the end of the last section, the interpretation of these correspondences in a dynamic context is not clear. In a strictly static framework we might visualize Walras' auctioneer calling out not only prices, but also a "reference" set of activities $(z, y)$. Thus the auctioneer would call out a $(z, y, p) \varepsilon$ w. On the basis of this, consumers would respond with $f^{i}(z, y, p)$ and producers with $r^{e}(z, y, p)$. In finding a solution (equilibrium) the auctioneer must find a point in $\mathrm{W},\left(z *, \mathrm{y}^{*}, \mathrm{p}^{*}\right)$ which has two properties:

$$
\text { i) } \Sigma z_{i}^{*}-\Sigma w^{i}\left(z *, y^{*}\right)-\Sigma \hat{y}_{e}^{*} \leqq 0 \text {, }
$$

and

$$
\text { ii) } z_{i}^{*} \in f^{i}\left(z^{*}, y^{*}, p^{*}\right) \text { and } y_{e}^{*} \in r^{e}\left(z^{*}, y^{*}, p^{*}\right) \text {. }
$$

Thus the auctioneer must call out both an equilibrium price vector and an associated set of equilibrium actions. It is not at all clear what the tatonnement stability properties of an equilibrium would be in a model with externalities.

In a truly dynamic framework things are much more complicated. One dynamic characterization of the model might be: $z_{i}^{t} \in f^{i}\left(z^{t-1}, y^{t-1}, p^{t}\right)$ where $\left(z^{t-1}, y^{t-1}\right)$ was the set of actions, actual or proposed at time $t-1$ by the various participants. However, if $\left(z^{t-1}, y^{t-1}\right)$ are the actual actions at time $t-1$, this means $\left(z^{t-1}, y^{t-1}\right)$ was technically feasible, which need not be the case. Thus the mechanics of a dynamic model with externalities are not obvious, but this paper will not attempt to solve this problem. However, the dynamic 
properties of such a model are very important because many policy prescriptions come from the analysis of the static model with externalities. This analysis is even more suspect because of the increased likelihood of multiple equilibria in models with externalities. This will be discussed later in this paper.

\section{B. The Attainable Set}

In this section we will examine the properties of the aggregate consumption-production possibilities set. As mentioned in our introduction, in a model slightly different from ours (he did not disaggregate production into input and output vectors), Osana established certain properties of this set. His main result established the compactness of this set. Our main contribution in this section is to establish its non-convexity. Since our model is closer in its assumptions to the one presented by Arrow and Hahn, we will need the following definitional material. We give all the assumptions sufficient to establish existence of equilibrium in our mode1, although we will not need all of the assumptions in our analysis.

Definition: We will call the vector $(z, y)$ resource feasible ( $r . f$. if $\Sigma x^{i}-\Sigma w^{i}(z, y)-\Sigma \hat{q}^{e} \leqq 0 . \quad\left(\operatorname{Recal1} z=\left(x^{1}, \ldots, x^{s}\right), y=\left(q^{1}, \ldots, q^{t}\right)\right)$ We will call a vector $(z, y)$ technologically feasible $(t . f$.$) if$ $\mathrm{x}^{i} \in \mathrm{X}^{\mathrm{i}}(\mathrm{z}, \mathrm{y}), \forall i$ and $\mathrm{y}_{\mathrm{e}} \in \mathrm{Y}^{\mathrm{e}}(\mathrm{z}, \mathrm{y}), \forall \mathrm{e}$. For a vector $(z, y)$ to be attainable for our model it must be both r.f. and t.f.

In this section we will establish under certain assumptions that the set of attainable actions in our model is bounded.

\section{Assumptions:}

7) For each e, $Y_{e}$ is such that $\hat{Y}_{e}$ is closed. ( $\hat{Y}_{e}$ will be convex by (4) since $\mathrm{Y}^{\mathrm{e}}$ is convex.)

8) Let $\hat{Y}=\sum_{e=1}^{t} \hat{Y}_{e}$. Then $\hat{Y} \cap \Omega^{n}=\{0\}$.

9) $\hat{\mathrm{Y}} \cap(-\hat{\mathrm{Y}}) \subset\{0\}$. 
Comments: That $\hat{\mathrm{Y}}_{\mathrm{e}}$ is closed is analogous to the usual assumption that the (fixed) net output set of a producer is closed. 9) is the usual irreversibility of production assumption. 8) and 9) are probably much stronger than necessary because $\hat{Y}$ contains much more than the set of $t . f$. net output vectors of the economy.

By 1) the $x^{i}(z, y)$ are uniformly bounded from below. Let $x^{-i}$ be such that $x^{i}<0$ and $x^{i}>\bar{x}^{i}, \forall x^{i} \in x^{i}(z, y), \forall i, \forall(z, y)$. Let $z_{i}=\left\{x^{i} \in R^{n} \mid x^{i} \geqq \bar{x}^{i}\right\}$. Then $x^{i}(z, y) \subset z_{i}, \forall(z, y), \forall i$. By 2$)$, for each $i \exists \bar{w}^{i}$ such that $w^{i}(z, y)<\bar{w}^{i}$, $\forall(z, y)$. Since $w^{i}(z, y) \geqq 0, \forall(z, y), \Sigma \bar{w}^{i} \geqq 0$ and $\Sigma \bar{w}^{i}>\Sigma w^{i}(z, y), \forall(z, y)$. Let $\Sigma \bar{w}^{i}=\bar{w}$.

\section{Definition}

$\left.\Sigma \hat{y}^{e} \leqq 0\right\}$

$$
\tilde{x}^{i}=\left\{x^{i} \in Z_{i} \mid \exists x^{j} \in z_{j} \text { and } y^{e} \in Y^{e} \text { such that } x^{i}+\sum_{j \neq i} x^{j}-\bar{w}-\right.
$$

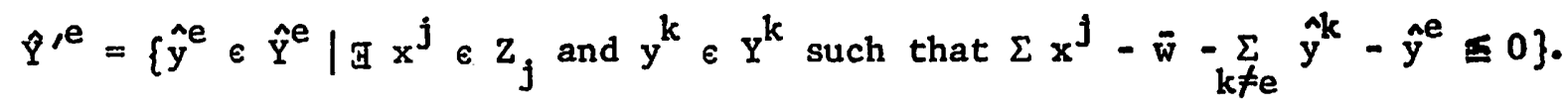

Since $0 \in \mathrm{Y}^{\mathrm{e}} \cdot \mathrm{Ve}$ and $\overline{\mathrm{w}}>\overline{\mathrm{X}}$, these sets are non-empty. $\left(\widetilde{\mathrm{X}}^{1}\right.$ contains the set of r.f. actions for $i$ and $\hat{Y}^{\prime e}$ contains the set of r.f. net outputs for $e$. )

Lemma 2: For economies satisfying 1$), 2), 6), 7), 8)$, and 9), $\hat{\mathrm{Y}}^{\text {(e }}$ and $\tilde{\mathrm{X}}^{1}$ are bounded, $\forall$ e,i.

Proof: The proof of this Lemma is identical to that found in the paper by Arrow and Debreu, ${ }^{5}$ and so we will not duplicate it here.

\section{Assumption}

10) For any $k>0$ let $B(k)=\left\{u \in R^{n}|| u_{h} \mid \leqq k, \forall h\right\}$ and for any $f>0$ let $C(j)=\left\{v \in R^{2 n}|| v_{h} \mid \leqq j, \forall h\right\}$. Then for any $G \subset \hat{Y}^{e}, G \subset B(k), G H \subset R^{2 n}$ such that $H \subset Y^{e}, G \subset \hat{H}$, and $H \subset C(j)$ for some $j>0$. 
What this assumption guarantees is that bounded net output sets are achievable by bounded sets of input-output vectors. Therefore, since $\hat{Y}^{\prime e}$ is bounded $\mathbb{H}^{\mathrm{a}}$ bounded set $\bar{Y}^{\prime e}$ such that $\hat{\bar{Y}}, e$ contains $\hat{Y}^{\prime e}$.

11) $x^{i}(z, y)$ has no satiation consumption, $\forall(z, y), \forall i$. (This means if $\bar{x}^{i} \in x^{i}(z, y)$ such that $(\bar{z}, y)(\geqq)_{i}\left(z^{\prime}, y\right), \forall z^{\prime}$ such that $i_{z}=i_{z}^{\prime}=I_{z}$, $\left.\bar{z}_{i}=\bar{x}^{-i}, z_{i}^{\prime} \in x^{i}(z, y)\right)$.

Notation: We will denote the economy constructed in section $A$ and satisfying assumptions 1) - 11) by the vector:

$$
E=\left\{\left(x^{i}(z, y), z_{i}, w^{i}(z, y),(\geqq)_{i}\right),\left(Y^{e}(z, y), Y^{e}\right),\left(d_{e}^{i}\right)\right\}
$$

We shall leave unproved the following Theorem, which is a simple variant of the Theorem proved in Arrow and Hahn.

Theorem III. The Economy $E$ which satisfies assumptions 1) - 11) has a competitive equilibrium. 6

We now will "compactify" the economy in order to proceed with our analysis.

Let $K$ be a cube in $R^{\mathfrak{n}}$ which contains each of the sets $\tilde{X}^{\mathbb{1}}, \hat{Y}^{\text {pe }}$ (defined in section $C$ ) in its Interior. Since $\forall(z, y)$ \& $\bar{x}^{i}(z, y)$ such that $\bar{x}^{i}(z, y) \ll w^{i}(z, y)$ and $0 \in \hat{y}^{e}, \forall e$, by construction, $w^{i}(z, y) \in$ Int $\tilde{x}^{1}$, $\forall(z, y)$, and thus $w^{i}(z, y) \in$ int $K, \forall(z, y)$. Since $0 \in \hat{Y}^{\prime e}, \forall e, 0 \in K$.

Let $\tilde{z}_{i}=z_{i} \cap k, \forall i$ and $\tilde{x}_{i}(z, y)=x^{i}(z, y) \cap K, \forall i, \forall(z, y)$. Then $\tilde{z}^{i}$ is non-empty convex and compact, $\tilde{x}^{i}(z, y)$ is non-empty convex and compact, and $\forall(z, y)$ H $\bar{x}^{i}(z, y) \in \tilde{x}^{i}(z, y)$ such that $\bar{x}^{i}(z, y) \ll w^{i}(z, y) \cdot \tilde{x}^{i}(z, y)$ is a continuous convex correspondence and $\tilde{x}^{\mathbb{I}}(z, y) \subset \tilde{z}_{i}, \forall(z, y)$. 
By 10), since $\hat{\mathrm{Y}}^{e} \cap \mathrm{K}$ is a compact set contained in $\hat{\mathrm{Y}}^{\mathrm{e}}$, a a bounded set $G^{e} \subset Y^{e}$ such that $\hat{Y}^{e} \cap K \subset \hat{G}^{e}$. Let $\tilde{Y}^{e}$ be such that $\tilde{Y}^{e} \supset G^{e}, \tilde{Y}^{e} \subset Y^{e}$, where $\tilde{Y}^{e}$ is convex and compact.

Let $\tilde{Y}^{e}(z, y)=Y^{e}(z, y) \cap \tilde{Y}^{e}$. Then $\tilde{Y}^{e}(z, y)$ is continuous, convex, compact and non-empty $($ by 6$))$ and $\tilde{Y}^{e}(z, y) \subset \tilde{Y}^{e}, \forall(z, y)$.

Now define a new economy, $\tilde{E}$, by

$$
\tilde{E}=\left\{\left(\tilde{X}^{i}(z, y), \tilde{z}_{i}, w^{i}(z, y),(\geqq)_{i}\right),\left(\tilde{Y}^{e}(z, y), \tilde{Y}^{e},\left(d_{e}^{1}\right)\right\} .\right.
$$

Let $\tilde{\mathrm{Y}}^{\mathrm{e}}$ and $\tilde{\mathrm{Z}}_{i}$ be as just defined, and let $\tilde{\mathrm{Y}}=\underset{\mathrm{e}=1}{\mathrm{X}} \tilde{\mathrm{Y}}_{e}, \mathrm{Z}={\underset{\mathrm{X}}{\mathrm{X}=1}}_{i}^{\mathrm{z}} \tilde{\mathrm{Z}}_{i}$, $\widetilde{W}=\tilde{Z} \times \tilde{Y} \times \tilde{P}$.

Consider the mapping $\Psi: \tilde{Z} \times \tilde{Y} \rightarrow \tilde{Z} \times \tilde{Y}$ where $\Psi(z, y)=\left[\mathbb{X}_{i=1}^{8} \tilde{X}^{1}(z, y)\right] \times$ $\left[\mathrm{e}_{\mathrm{e}=1}^{t} \tilde{Y}^{i}(\mathrm{z}, \mathrm{y})\right]$. Under the previous assumptions $\Psi$ is a continuous convex-valued correspondence (since $\tilde{W}$ is compact) and $\tilde{Z} \times \tilde{Y}$ is compact and convex. Thus by Kakutani's theorem $\Psi$ has a fixed point. (Notice that we only need $x^{i}(z, y)$ and $\mathrm{Y}^{\mathrm{e}}(\mathrm{z}, \mathrm{y})$ to be upper semi-continuous and convex-valued or only continuous to make

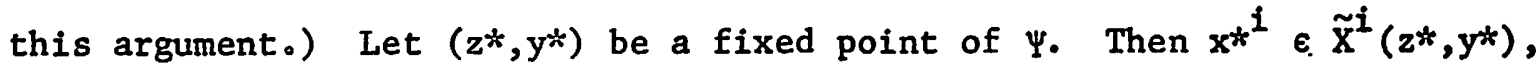
$\forall i$ and $q^{*}{ }^{e} \in \tilde{Y}^{e}\left(z^{*}, y^{*}\right), \forall e_{0} \quad$ Since $\tilde{X}^{i}(z, y) \subset X^{i}(z, y), \forall i$ and $\tilde{Y}^{e}(z, y) \subset Y^{e}(z, y)$, $\forall e$, we see that a fixed point of $\Psi$ is a vector of technologically feasible (t.f.) actions.

Let $F$ be the set of fixed points of $\Psi$. Then $F$ is the set of $t . f$. actions in $\tilde{\mathrm{E}}$. 
Lemma 3: F is compact.

Proof: $F$ is clearly bounded. ( $\tilde{Z}$ and $\tilde{Y}$ are bounded.) Suppose $F$ is not closed. Then there exists a sequence $\left\{\left(z^{n}, y^{n}\right)\right\}$ such that $\left(z^{n}, y^{n}\right) \rightarrow(z, y)$, $\left(z^{n}, y^{n}\right) e \Psi\left(z^{n}, y^{n}\right), \forall n$ but $(z, y) \& \Psi(z, y)$. But this is a contradiction of $\Psi$ upper semi-continuous. Thus $\mathrm{F}$ is closed. (As we will show, F will not in general be convex.)

Now consider the mapping $\xi: \tilde{Z} \times \tilde{Y} \rightarrow R^{n}$ where $\xi(z, y)=z-\Sigma w^{1}(z, y)-$ $\Sigma \hat{q}^{e}$. Since $w^{i}(z, y)$ is continuous, $V i, \xi(z, y)$ is a continuous function, and furthermore 0 is contained in the range of $\xi$ because $w^{i}(z, y) \in \tilde{z}_{i}, \forall i$ and $0 \in \tilde{Y}^{e}, \forall e . \quad$ Let $B=\{(z, y) \notin \tilde{Z} \times \tilde{Y} \mid \xi(z, y) \leqq 0\}$. Then $B$ is non-empty and compact since $\xi$ is continuous.

Let $A=B \cap F$. Then $A$ is the set of attainable actions in $E$. A is compact since $B$ and $F$ are. We must show that $A$ is non-empty.

\section{Lemma 4: A is non-empty.}

Proof: Consider the mapping $\alpha: \tilde{z} \rightarrow \tilde{z}$ where $\alpha(z)={\underset{x}{x=1}}_{i} w^{i}(z, 0)$. By the continuity of the $w^{i}(z$,$) , we have that \alpha(z)$ is continuous and since $\tilde{\mathrm{Z}}$ is compact and convex $\alpha$ has a fixed point. Let $\overline{\mathrm{z}}$ be a fixed point of $\alpha$. Then $(\bar{z}, 0) \in F$ because $0 \in \tilde{Y}^{e}(z, y), \forall(z, y)$ and $w^{i}(z, y) \in \tilde{X}^{i}(z, y), \forall(z, y)$. Also, $(\bar{z}, 0) \in B$ because by definition of $\alpha, \underset{i=1}{s} w^{i}(\bar{z}, 0)=\bar{z}$. Thus $(\bar{z}, 0) \in A$. As mentioned earlier, A is the set of attainable actions for the economy $\mathrm{E}$ and as we have shown, $\mathrm{A}$ is non-empty and compact.

Given Lemma 4 and our assumptions on the preferences $(\geqq)_{1}$, it is easy to establish the following Theorem. 
Theorem IV: The economy $\mathrm{E}$ has a Pareto Optimum.

The assumptions we have used to guarantee the existence of an optimum are stronger than the corresponding assumptions used in the treatment of the problem for economies without externalities. For example, we needed to have the $\tilde{\mathrm{X}}^{\mathrm{i}}(\mathrm{z}, \mathrm{y})$ to be convex, which is not necessary in the usual case. As indicated earlier, the assumption we used in showing $\Psi$ has a fixed point and $F$ is compact only requires that $\tilde{X}^{i}(z, y)$ and $\tilde{Y}^{e}(z, y)$ are convex and u.s.c.

$F$ is the "compactified" disaggregated consumption-production set for an economy with externalities. F of course is different from the disaggregated consumption-production set of an economy without externalities. In such an economy this set is just a simple Cartesian production of the convex individual consumption and production sets. This is not true of $F$, where different agents' actions are connected by the joint feasibility constraint. F also is not generally convex.

To see this, recall that $F$ is the set of fixed points of the mapping $\Psi: \tilde{Z} \times \tilde{Y} \rightarrow \tilde{Z} \times \tilde{Y}$, where $\Psi(z, y)=\left(\underset{i=1}{S} \tilde{X}^{i}(z, y)\right) \times\left(\underset{e=1}{t} \tilde{Y}^{e}(z, y)\right)$. Define the "graph" of $\tilde{X}^{i}(z, y), G^{i}$, by $G^{i}=\left\{(z, y) \in \tilde{z} x \tilde{y} \mid z_{i}=x^{i} \in \tilde{X}^{1}(z, y)\right\}$ and the "graph" of $\tilde{Y}^{e}(z, y), G^{e}$ by $G^{e}=\left\{(z, y) \in \tilde{Z} \times\left.\tilde{Y}\right|_{y}=q^{e} \in \tilde{Y}^{e}(z, y)\right\}$.

The "graph" is analogous to the conventionally defined graph of a correspondence. For example if $f: R \rightarrow R$, the graph of $f, G_{f}=$ $\left\{(x, y) \in R^{2} \mid y=f(x)\right\}$. The only difference between our "graph" and the conventionally defined graph, is that the range of the correspondence 18 not in general located in the last coordinate for the "graph." Recall that $F=\left\{(z, y) \mid z_{i}=x^{i} \in \tilde{X}^{i}(z, y), \forall i\right.$ and $\left.y_{e}=q^{e} \in \tilde{Y}^{e}(z, y), \forall e\right\}$. Therefore, $F$ is just the intersection of the "graphs" of the $\tilde{X}^{i}(z, y)^{\prime} s$ and $\tilde{Y}^{e}(z, y)^{\prime} s$, i.e., 


$$
F=\left(\bigcap_{i=1}^{s} G^{i}\right) \cap\left(\bigcap_{e=1}^{t} G^{e}\right)
$$

To see this, let $(\mathrm{z}, \mathrm{y}) \in\left(\bigcap_{i=1}^{\mathrm{s}} \mathrm{G}^{i}\right) \cap\left(\bigcap_{\mathrm{e}=1}^{t} \mathrm{G}^{\mathrm{e}}\right)$. Then by definition of $\mathrm{G}^{1}$ and $G^{e}$, we have $z_{i}=x^{i} \in \tilde{X}^{i}(z, y), \forall i$ and $y_{e}=q^{e} \in \tilde{Y}^{e}(z, y), \forall e$.

Since $F$ is non-empty, this intersection of sets is non-empty. Now $G^{i}$ is the "graph" of a continuous convex-valued correspondence. Therefore $G^{i}$ will be compact. However, $G^{i}$ will not in general be convex. To see this, consider for exanple the function. $f:[0,1] \rightarrow[0,1], f(x)=x^{2}$. $f$ is certainly continuous and convex valued. But the graph of $f, G_{f}=$ $\left\{(x, y) \mid y=x^{2}\right\}$ is certainly not convex. In fact, only if $f$ is linear will $G_{f}$ be convex! Therefore the $G^{i}$ 's and $G^{e}$ 's will not generally be convex. But since $F$ is the intersection of the $G^{i^{\prime}} s$ and $G^{e^{\prime}} s, F$ will also generally not be convex. This will also be true of the set of attainable actions, $A=\left\{(z, y) \in F \mid \Sigma x^{i}(z, y)-\Sigma w^{i}(z, y)-\Sigma \hat{q}^{e} \leqq 0\right\}$. Thus we have seen that the underlying technologically feasible set of actions of an econony with externalities will usially not be convex. One might ask whether this underlying t.f. set could in sone cases arise from a non-convex economy without externalities (e.g., increasing returns). The answer to this is no, because although the t.f. set of actions of a non-convex econouny will usually be nonconvex, it will still be the simple Cartesian product of sets, which wlll not be the case in an econony with externalities.

\section{c: The Aggregate Excess Demand Correspondence}

Next, we shall establish the existence and propertles of what we shall call the aggregate excess demand correspondence for our economy. In this exercise we invoke the assumptions of Parts $A$ and $B$. 
Let $\hat{f}^{1}(z, y, p)$ denote the excess denand corresponfence of consumer 1 relative to the "compactified" econony $\tilde{E}$, and let $\tilde{r}^{e}(z, y, p)$ denote the supply correspondence of producer e relative to the "conpactified" econoray $\tilde{E}$.

Consider the corsspondence $\mathrm{K}_{\mathrm{p}}: \tilde{\mathrm{Z}} \times \tilde{\mathrm{Y}} \rightarrow \tilde{\mathrm{Z}} \times \tilde{\mathrm{Y}}$, where $\mathrm{K}_{\mathrm{p}}(\mathrm{z}, \mathrm{y})=$ $\left(\stackrel{\mathrm{X}}{\mathrm{X}}_{i=1}^{\mathrm{f}} \tilde{\mathrm{f}}^{\mathrm{I}}(\mathrm{z}, \mathrm{y}, \mathrm{p})\right) \times\left(\mathrm{e}_{\mathrm{X}}^{\mathrm{t}} \tilde{\mathrm{r}}^{\mathrm{e}}(\mathrm{z}, \mathrm{y}, \mathrm{p})\right)$. Since the assumptions of Parts $A$ and $B$ are in force and $\tilde{W}$ is compact, $\tilde{f}^{1}(z, y, p)$ is $u . s . c$. and convex-valued, $V 1$ and $\tilde{r}^{e}(z, y, p)$ is u.s.c. and convex-valued, $\forall e$. Therefore $k_{p}(z, y)$ will be u.s.c. and convex-valued, $\forall p \in P$, and by Kakutani's Theorem will have a fixed point. ( $\tilde{Z} \times \tilde{Y}$ is compact and convex). Let $d(p)$ be the set of fixed points of $K_{p}$. Then $d(p)$ is the set of consistent demands and supplies at $p$. To see this, let $(z, y)$ be fixed point of $k_{p}$. This means that $(z, y) \in\left({\underset{i=1}{X}}_{\tilde{f}^{i}}^{s}(z, y, p)\right) \times\left(\mathbb{X}_{e=1}^{t} \tilde{r}^{e}(z, y, p)\right), i . e ., z_{i}=x^{1} \in \tilde{f}^{1}(z, y, p), \forall i$ and $y_{e}=q^{e} \varepsilon \cdot \tilde{r}^{e}(z, y, p), \forall e \cdot d(p)$ therefore is the set of "stable" demands and supplies at $p$ in the sense that no participant has any desire to change his action if $d(p)$ is the set of actions at $p$.

$$
\begin{aligned}
& d(p) \text { is a correspondence, } d(p): p \rightarrow \tilde{Z} x \tilde{Y} \text {. Let } \\
& \quad \tilde{f}_{\tilde{f}^{i}}(p)=\left\{(z, y) \mid z_{i}=x^{i} \in \tilde{\mathbb{f}}^{i}(z, y, p)\right\}
\end{aligned}
$$

and

$$
G_{\tilde{r} e^{e}}(p)=\left\{\left.(z, y)\right|_{e}=q^{e} \in \tilde{r}^{e}(z, y, p)\right\}
$$

$G \tilde{f}^{i}(p)$ is just the "graph" of the correspondence $\tilde{f}^{\mathcal{I}}(z, y, p)$, where $p$ is kept fixed; for example if we define a new correspondence, $k_{\bar{p}}^{i}(z, y)=\tilde{f}^{i}(z, y, \tilde{p})$, then $G_{\tilde{f}^{i}}(\bar{p})$ is just the "graph" of $k_{\bar{p}}^{i}(z, y)$. To determine the topological properties of $d(p)$ it is useful to note that

$$
d(p)=\left(\bigcap_{i} G \tilde{f}_{i}(p)\right) \cap\left(\bigcap_{e} G_{\tilde{r}^{e}}(p)\right),
$$

and we can therefore determine the properties of $d(p)$ by determining the properties of $G_{\tilde{f}^{i}}(p)$ and $G_{\tilde{r}^{e}}(p)$ 
Lemma 5: $d(p)$ is an u.s.c. correspondence.

Proof: First we must show $G_{\tilde{f}^{i}}(p)$ and $G_{\tilde{r}^{e}}(p)$ are $u .8 . c_{.}:$, Let $p^{\mathfrak{n}} \rightarrow p, p^{\mathfrak{n}}$ and $p \in P$, and $\left(z^{n}, y^{n}\right) \in G \tilde{f}^{i}\left(p^{n}\right), \forall n$, and $\left(z^{n}, y^{n}\right) \rightarrow(z, y)$. We must show that $(z, y) \in G \tilde{f}^{i}(p) \cdot\left(z^{n}, y^{n}\right) \in G \tilde{f}^{i}\left(p^{n}\right), \forall n$ implies $\left(z^{n}, y^{n}\right) \in \tilde{z} x \tilde{Y}, \forall n$ and $z_{i}^{n}=\left(x^{i}\right)^{n} \ddot{\epsilon} \tilde{f}^{i}\left(z^{n}, y^{n}, p^{n}\right)$. But $\tilde{f}^{i}(z, y, p)$ is u.s.c., and therefore $\left(z^{n}, y^{n}, p^{n}\right) \rightarrow(z, y, p)$, and $z_{i}^{n}=\left(x^{1}\right)^{n} \in \tilde{f}^{i}\left(z^{n}, y^{n}, p^{n}\right), \forall n$ implies that $\lim \left(x^{i}\right)^{n}=z_{i}=x^{i} \in \tilde{f}^{i}(z, y, p)$. Therefore $G \tilde{f}_{i}(p)$ is u.s.c., and in the same manner we can show that $G_{\tilde{r} e}(p)$ is u.s.c. Since $d(p)$ is the (nonempty) intersection of u.s.c. correspondences, $d(p)$ is u.s.c.

As was shown for the set $F$, since $d(p)$ is the intersection of the "graphs" of correspondences, d(p) will generally not be convex valued. Let

$$
\begin{aligned}
D(p)=\left\{\Sigma x^{i}-\Sigma w^{i}(z, y)-\Sigma \hat{q}^{e} \mid(z, y) \in d(p)\right. & \\
& \left.z=\left(x^{1}, \ldots, x^{s}\right) ; y=\left(q^{1}, \ldots, q^{t}\right)\right\}
\end{aligned}
$$

Then $D(p)$ may properly be called the Aggregate Excess Demand Correspondence

for the compactified economy $\tilde{E}$, because $D(p)$ is the set of consistent aggregate excess demands at $p . D(p)$ is completely analogous to the aggregate excess demand correspondence defined in an economy without externalities. ("compactified" in the usual manner).

Since $d(p)$ is $u . s . c$. but not generally convex $D(p)$ w111 also have these properties. For the usual general equilibrium model without externalities treated in the existence 1iterature the aggregate excess demand correspondence is u.s.c. and convex. 
D. Some Examples

a) Consider the following example of a 2-person, 2-good pure exchange economy .

Notation: $x_{1}^{A}$ is consumption of good 1 by Mr. A.

$\mathrm{x}_{2}^{\mathrm{B}}$ is consumption of good 2 by Mr. B, etc.

Data:

Utility function of Mr. A: $U^{A}\left(x_{1}^{A}, x_{2}^{A} ; x_{1}^{B}, x_{2}^{B}\right)=\left(x_{1}^{A}+x_{1}^{B}\right) \cdot\left(x_{2}^{A}\right)^{3}$

Endowment of Mr. A: $w_{1}^{A}=w_{2}^{A}=4$

Utility function of $M r \cdot B: U^{B}\left(x_{1}^{B}, x_{2}^{B} ; x_{1}^{A}, x_{2}^{A}\right)=\left(x_{1}^{B}-x_{1}^{A^{2}}+6 x_{1}^{A}\right) x_{2}^{B}$

Endowment of Mr. B: $\underset{w_{1}}{B}=\underset{w_{2}}{B}=4$

Good 1 is numeraire so $p_{1}=1, p_{2}=p$. For points of interior maximum $\left(x_{1}^{A}, x_{1}^{B}>0\right)$ the demand functions for good 1 are:

i) $x_{1}^{A}=1+p-\frac{3}{4} x_{1}^{B} \quad\left(x_{1}^{A}, x_{1}^{B} \geqq 0\right)$

ii) $x_{1}^{B}=2+2 p+\frac{x_{1}^{A^{2}}-6 x_{1}^{A}}{2}\left(x_{1}^{A}, x_{1}^{B} \geqq 0\right)$

To get the set of consistent demands at $p$, we solve (i) and (1i) simultaneously.

Substituting (ii) into (i) gives us:

$$
x_{1}^{A}=1+p-\frac{3}{4}\left[2+2 p+\frac{x_{1}^{A^{2}}-6 x_{1}^{A}}{2}\right] \text {. }
$$

Solving for $x_{1}^{A}$ :

$$
3 x_{1}^{A}-10 x_{1}^{A}+4 p+4=0
$$

and solving using the quadratic formula, we have:

$$
x_{1}^{A}=\frac{10 \pm \sqrt{100-12(4 p+4)}}{6}
$$


Thus, for example, for $p=1$, we have the set of consistent demands

$$
\left[x_{1}^{A}=2 ; \quad x_{1}^{B}=0\right]
$$

or

$$
\left[x_{1}^{A}=4 / 3 ; x_{1}^{B}=8 / 9\right]
$$

Denote the excess demands by $z_{1}^{A}$, etc. (Then $z_{1}^{A}=x_{1}^{A}-4, z_{2}^{B}=x_{2}^{B}-4$; etc.). Then the excess demand functions are:

$$
\begin{aligned}
& \text { iv) } \mathrm{z}_{1}^{\mathrm{A}}=\mathrm{p}-6-\frac{3}{4} \mathrm{z}_{1}^{\mathrm{B}} \\
& \text { v) } \mathrm{z}_{1}^{\mathrm{B}}=2 \mathrm{p}-6+\frac{\mathrm{z}_{1}^{\mathrm{A}^{2}}-2 \mathrm{z}_{1}^{\mathrm{A}}}{2}
\end{aligned}
$$

These excess demand functions are valid only for points of interior maximum. For example, in (iii) consider $p=3$. For $p=3$ (iii) has no real solutions, i.e., there is no set of consistent demands given demand functions ( 1 ) and (ii). The problem here is that (i) and (ii) are not valid for $p=3$ because the solutions of the consumer maximum problems are not interior solutions at $p=3$. 
The "global" demand functions are:

$$
\begin{aligned}
& \text { i') } x_{1}^{A}=\left\{\begin{array}{l}
1+p-\frac{3}{4} x_{1}^{B}, \text { if } 1+p-\frac{3}{4} x_{1}^{B} \geqq 0 \\
0, \text { otherwise }
\end{array}\right\} x_{1}^{B} \geqq 0 \\
& \text { ii') } x_{1}^{B}=\left\{\begin{array}{l}
2+2 p+\frac{x_{1}^{A^{2}}-6 x_{1}^{A}}{2}, \text { if } 2+2 p+\frac{x_{1}^{A^{2}}-6 x_{1}^{A}}{2} \geqq 0 \\
0, \text { otherwise }
\end{array}\right\} x_{1}^{A} \geqq 0
\end{aligned}
$$

This gives us global versions of (iv) and (v):

$$
\begin{aligned}
& \text { iv') } z_{1}^{A}=\left\{\begin{array}{l}
p-6-\frac{3}{4} z_{1}^{B}, \text { if } p-6-\frac{3}{4} z_{1}^{B} \geqq-4 \\
-4, \text { otherwise }
\end{array}\right\} z_{1}^{B} \geqq-4 \\
& \left.v^{\prime}\right) z_{1}^{B}=\left\{\begin{array}{l}
2 p-6+\frac{z_{1}^{A^{2}}+2 z_{1}^{A}}{2}, \text { if } 2 p-6+\frac{z_{1}^{A^{2}}+2 z_{1}^{A}}{2} \\
-4, \text { otherwise }
\end{array}\right\} z_{1}^{A} \geqq-4
\end{aligned}
$$

In the following diagram we depict excess demands of $A$ and $B$ in the following manner:

$z_{1}^{B}\left(1 ; z_{1}^{A}\right)$ for example is the excess demand of $B$ for good 1 as a function of $z_{1}^{A}$ for $p=1, \quad z_{1}^{B}\left(3 ; z_{1}^{A}\right)$ is the excess demand of $B$ for good 1 as a function of $z_{1}^{A}$ for $p=3$, etc.

Points marked.; are points of consistent excess demand for $p=1$. They are the points: $(-2,4),(-8 / 3,-28 / 9),(-4,0)$. The point marked $O$ is the point of consistent excess demand for $p=3$. The 1 ine $z_{1}^{A}+z_{1}^{B}=0$ represents the locus of possible equilibria. If $\left\{z^{A}\left(\bar{p} ; z_{1}^{B}\right), z_{1}^{B}\right\} \cap\left\{z_{1}^{A}, z_{1}^{B}\left(\bar{p} ; z_{1}^{A}\right)\right\}$ contains a point on this line for some $\overline{\mathrm{p}}$, then $\overline{\mathrm{p}}$ is an equilibrium price. Thus, since the point marked 0 has this property, $p=3$ is an equilibrium, since it is a point of conststent excess demands for good 1, and the sum of the excess demands for good 1 is zero. By Walras Law therefore the excess demand for good 2 will also be zero. 


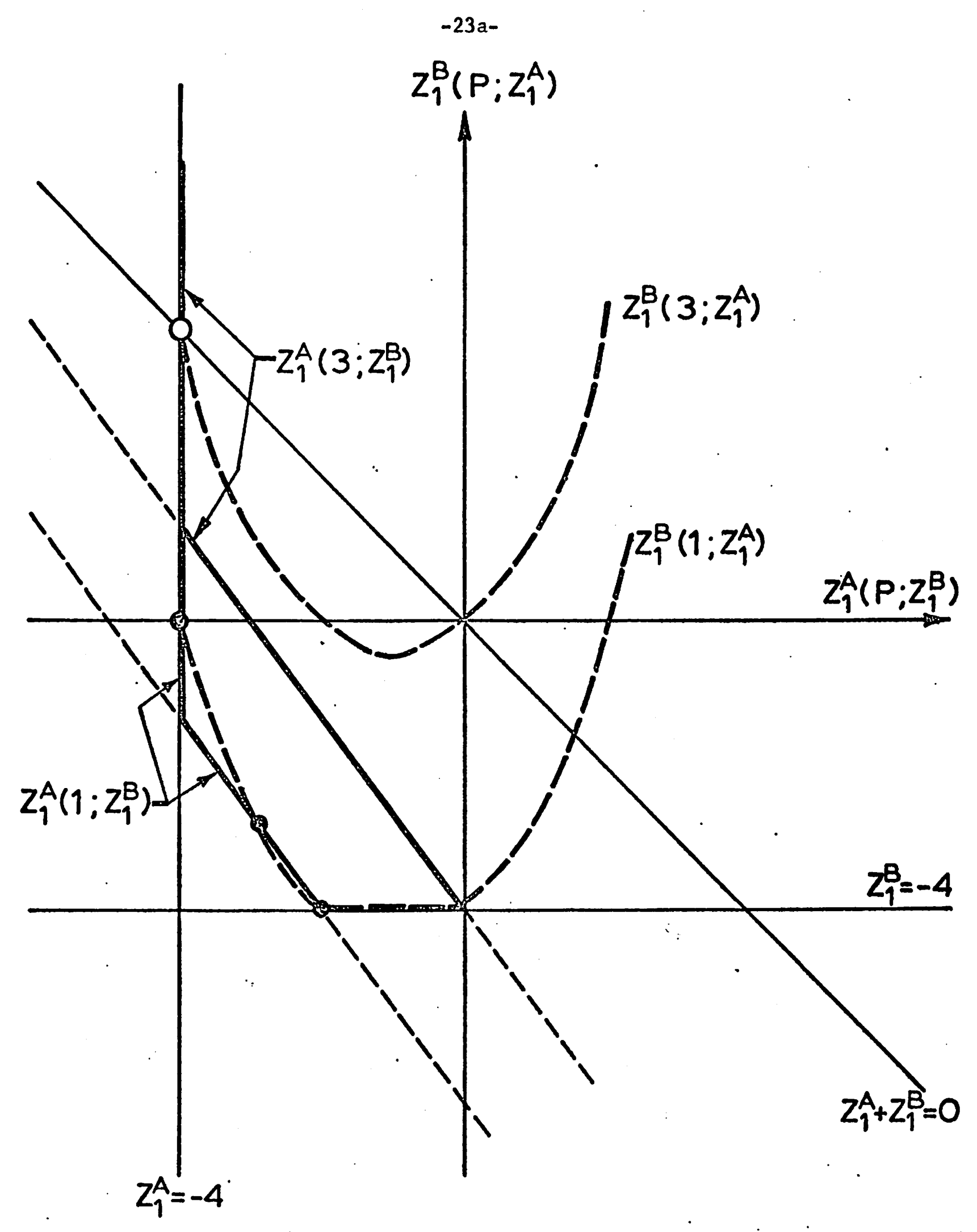


We see from the diagram that for this exrample the Aggregate Excess Demand Correspondence is clearly not convex-valued at $p=1$.

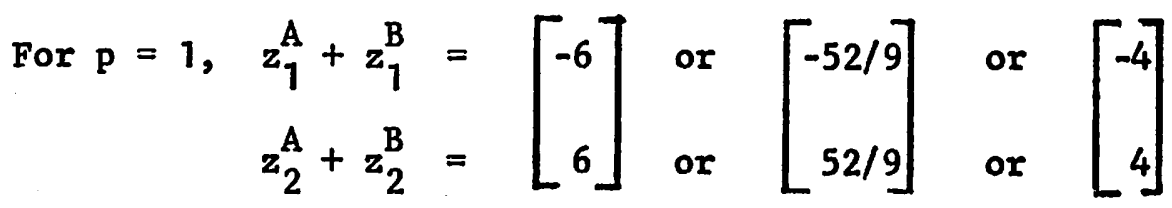

Therefore $\left.d(p)\right|_{p=1}=\{(-6,6),(-52 / 9,52 / 9),(-4,4)\}$

For $0<13 / 12, D(p)$ will take on three distinct values for each $p$. For $p=13 / 12, D(p)$ will take on two distinct values. For $p>13 / 12, D(p)$ will be single valued.

b) With externalities, the possibility of multiple equilibris is greater. Consider the following example.

Data:

Utility function of Mr. A: $\left(x_{1}^{A}+x_{1}^{B}\right)^{a}\left(x_{1}^{A}+x_{2}^{B}\right)^{1-a}$ where a $=11 / 40$

Endowment of Mr. A: $w_{1}^{A}=33 / 8, w_{2}^{A}=7 / 8$

Utility function of Mr. B: $\left(x_{1}^{B}-x_{1}^{A^{2}}+6 x_{1}^{A}\right) x_{2}^{B}$

Endowment of Mr. B: $w_{1}^{B}=0, w_{2}^{B}=10$

The demand functions for good 1 for $A$ and $B$ are:

$$
\begin{aligned}
& x_{1}^{A}= \begin{cases}\left(I^{A}+I^{B}\right)-x_{1}^{B}, & \text { if } \geqq 0 \text { and } \leqq I^{A} \\
0, & \text { if }<0 \\
I^{A}, & \text { if }>I^{A}\end{cases} \\
& x_{1}^{B}= \begin{cases}I^{B} / 2+\frac{x_{1}^{A}-6 x_{1}^{A}}{2}, & \text { if } \geqq 0 \text { and } \leqq I^{2} \\
0, & \text { if }<0 \\
I^{B}, & \text { if }>I^{B}\end{cases}
\end{aligned}
$$




\begin{abstract}
E. Summary
We have established the compactness and probable non-convexity set of attainable actions for general equilibrium economies with externalities. We have also defined the aggregate excess demand correspondence for such economies in a manner which allows direct comparison with the aggregate excess demand correspondence for economies without externalities. Our analys is established the upper semi-continuity of this correspondence and the likelihood of its non-convexity. These results argue that in a sense, externalities introduce non-convexities into the usual static general equilibrium model.
\end{abstract}




\section{Footnotes}

* The author expresses gratitude to Paul Samuelson, Robert Solow Franklin Fisher, Duncan Foley, and Peter Diamond for helpful discussions on an earlier version of this paper.

${ }^{1}$ Osana, H., "On the Boundedness of an Economy with Externalities," Review of Economic Studies, July 1973.

${ }^{2}$ Arrow, K. J., and Hahn, F. H., General Competitive Analysis, Holden-Day, Inc., 1971, pp. 132-136.

${ }^{3}$ Scheffman, D. T., Two Essays in Economic Theory, Unpublished Doctoral Dissertation, M.I.T., 1971.

${ }^{4}$ Debreu, G., "New Concepts and Techniques in Equilibrium Analysis," International Economic Review, 3, 257-273, 1962. pp. 55-59.

5 Debreu, G., Theory of Value, John Wiley and Sons, Inc., 1959,

${ }^{6}$ Arrow, K. J., and Debreu, G., "Existence of and Equilibrium for a Competitive Economy," Econometrica, 22, 265-290, 1954, pp. 276-277.

${ }^{7}$ Given Theorems I and II and Lemma 2 it is easy to construct a proof similar to that used by Debreu in Theory of Value. Let $M(z, y)=\left\{p^{\prime} \in P \mid p^{\prime} . \quad\left[\Sigma x^{i}(z, y)-\Sigma w^{i}(z, y)-\Sigma \hat{q}^{e}\right] \geqq p \cdot\left[\Sigma x^{i}-\Sigma w^{i}(z, y)-\Sigma \hat{q}^{e}\right]\right.$ for all $\mathrm{p} \in \mathrm{P}\}$. Then, to get the necessary fixed point, use the mapping: $\phi: \quad \widetilde{W} \rightarrow \widetilde{W}$, where $\phi(z, y, p)={\underset{i=1}{X}}_{i} f^{i}(z, y, p) \times \underset{e=1}{x} r^{e}(z, y, p) \times M(z, y)$, where $\widetilde{W}$ is as defined in the next material. This gives the existence Theorem for the "compactified" economy. In a manner similar to Debreu it can then be shown that this also gives an equilibrium for the original economy. 